\title{
A Bayesian Hierarchical Spatio-Temporal Model for Extreme Temperatures in Extremadura (Spain) Simulated by a Regional Climate Model
}

\section{Jose Agustin Garcia}

Universidad de Extremadura

Francisco Javier Acero ( $\nabla$ fjacero@unex.es )

Universidad de Extremadura https://orcid.org/0000-0003-2073-8232

Javier Portero

Universidad de Extremadura

\section{Research Article}

Keywords: Extreme Temperature, Regional Climate Model, GEV Distribution, Bayesian Hierarchical Model

Posted Date: December 30th, 2021

DOl: https://doi.org/10.21203/rs.3.rs-1178315/v1

License: (c) (i) This work is licensed under a Creative Commons Attribution 4.0 International License.

Read Full License 
Springer Nature 2021 LATEX template 


\title{
A Bayesian hierarchical spatio-temporal model for extreme temperatures in Extremadura (Spain) simulated by a Regional Climate Model
}

\author{
José Agustín García ${ }^{1,2 \dagger}$, Francisco Javier Acero ${ }^{1,2^{*} \dagger}$ \\ and Javier Portero ${ }^{1 \dagger}$ \\ $1^{*}$ Departamento de Física, Universidad de Extremadura, Avd. \\ Elvas s/n, Badajoz, 06006, Spain. \\ ${ }^{2}$ Instituto Universitario de Investigación del Agua, Cambio \\ Climático y Sostenibilidad (IACYS), Universidad de \\ Extremadura, Avd. Elvas s/n, Badajoz, 06006, State, Spain.

\begin{abstract}
*Corresponding author(s). E-mail(s): fjacero@unex.es; Contributing authors: agustin@unex.es; jportero@unex.es; $\dagger$ These authors contributed equally to this work.
\end{abstract}

\begin{abstract}
A statistical study was made of the temporal trend in extreme temperatures in the region of Extremadura (Spain) during the period 1981-2015 using a Regional Climate Model. For this purpose, a dataset of extreme temperature was obtained from the Weather Research and Forecating (WRF) Regional Climate Model. This dataset was then subjected to a statistical study using a Bayesian hierarchical spatiotemporal model with a Generalized Extreme Value (GEV) parametrization of the extreme data. The Bayesian model was implemented in a Markov chain Monte Carlo framework that allows the posterior distribution of the parameters that intervene in the model to be estimated. The role of the altitude dependence of the temperature was considered in the proposed model. The results for the spatial-trend parameter lend confidence to the model since they are consistent with the dry adiabatic gradient. Furthermore, the statistical model showed a slight negative trend for the location parameter. This unexpected result may be due to the internal and modeling uncertainties
\end{abstract}


in the WRF model. The shape parameter was negative, meaning that there is an upper bound for extreme temperatures in the model.

Keywords: Extreme Temperature, Regional Climate Model, GEV Distribution, Bayesian Hierarchical Model

\section{Introduction}

It has now become quite clear that the emission into the atmosphere of huge amounts of $\mathrm{CO}_{2}$ from the combustion of fossil fuels by humankind, together with other changes such as deforestation and intensive agriculture, is altering the climate, with rising temperatures over most of the planet [1]. One concern for society and its policymakers is that higher average temperatures may enhance the risk of extreme events such as heat waves. These are associated with effects that can be lethal for humans, and, even below lethality, can have adverse impacts on their livelihoods [2]. Examples of such heat waves were those that occurred in Southern Europe in 2003 [3] and Russia in 2010 [4].

The best tools with which to project the future evolution of these extreme events are global climate models (GCMs) that take account of forcing from greenhouse gas emissions. At present however, these models are too coarse to properly represent atmospheric properties such as temperature or precipitation at the local scale which is needed for adaptive planning and policy making. This issue is usually addressed by downscaling the GCMs using Regional Climate Models (RCMs). An RCM is a limited-area weather model embedded in a GCM, or a higher resolution reanalysis that takes inhomogeneities of the Earth's surface into account as well as other properties not covered by the coarser GCMs. Obviously, if an RCM is to be used to study the future state of the climate then it must describe reasonably well the current state. The variable on which the present study will focus is that of extreme temperatures.

There are two main ways to approach the study of extreme meteorological and climate events. One is to use extreme indices such as some high percentile of the probability distribution function [5-7]. The other is to apply the branch of statistics known as extreme value theory (EVT). An important advantage of the latter is that the technique is more informative than that of the extreme indices since, by obtaining the parameters of the distribution function, one can estimate the likelihood of as-yet unobserved events - the 100-year return level for example. This feature is widely used for engineering design purposes. The present work in particular applies EVT in order to get a climatological picture of extreme temperature events through estimating the parameters of the corresponding extreme value distribution. Given the spatial character of temperature as a variable, it seemed appropriate to use the theory of spatial extremes to study it. Three approaches have typically been used to address the problem of spatial extremes - max-stable random fields, copulas, and Bayesian hierarchical models [8] - and it is this last approach we shall take in the present 
work. One of the more important benefits of using a spatial extremes theory instead of modeling each observatory individually is the increased precision when estimating the parameters of the statistical distribution. Indeed, one of the difficulties one faces in a statistical study of extreme data is that, by their very nature, extreme data are 'rare', i.e., there are usually so few data that one must expect to get a large uncertainty in the parameters that describe the tail of the statistical distribution. One way to mitigate the consequences of this lack of data and to increase the precision of the parameter estimates is to trade space for time, pooling information from different observatories (see, for example, [9-11]). Also, as pointed out by Renard [12], a spatial theory allows the parameters of the extreme distribution to be estimated at an ungauged or poorly gauged site. This important advantage allows one to address the so-called change of support problem in spatial statistics (see [13], [14]). This problem arises when one tries to compare data coming from sources at different spatial scales. In our case, we want to compare the extreme value distribution obtained from a model defined at a grid-cell scale with that obtained at a meteorological observatory defined at a local scale. Moreover, the use of Bayesian statistics allows one to account properly for the uncertainties that naturally arise when modeling meteorological phenomena (see [15]).

Most published studies dealing with extreme weather and climate events obtained from GCM/RCM models used extreme indices. Far fewer have used Extreme Value Theory (EVT). As one example of applying EVT, [16] made a study of extreme temperature and precipitation in a GCM for the period 19902100, considering several scenarios using a GEV distribution. Also, [17] made an analysis of extreme precipitation in Germany using a non-homogeneous Poisson point process to determine the GEV parameters. Both cases, however, fitted the statistical model to the results obtained at each grid point individually. On the other hand, [11] used a spatial hierarchical model in a study of extreme precipitation obtained from six different RCMs. That study used a multivariate intrinsic autoregressive (IAR) spatial model - a kind of Gaussian Markov Random Field model. While this type of model is computationally well suited to fitting the gridded output of a climate model, it is less suitable for making a prediction at a 'non-observed' site. A model closer to the one used in the present work is that of [18] who studied extreme minimum temperatures obtained in Sweden from an RCM. The main problem we have found in this paper is that the analysis does not take the altitude dependence of the temperature into account, a dependence that is now included in the present model.

The objective of the present study was therefore twofold - firstly, to simulate the present climate in our region by means of an RCM, specifically, the WRF V4.0 model using ERA Interim reanalysis boundary conditions, secondly, to produce a statistical model which can be used to characterize summer extreme temperatures produced by the RCM for the region of Extremadura (Spain). This statistical model will then be used to assess whether the model can correctly describe the region's observed extreme temperatures. 
The paper is organized as follows. The data used are described in Section 2 , the details of the statistical model are presented in Section 3, and the results in Section 4. Finally, some conclusions are discussed in Section 5.

\section{Data}

The temperature data used come from a simulation of the climate using the community WRF model (version 4.0.1) [19] forced with ERA Interim [20] reanalysis. The period for the simulation is 1980-2015. The simulation used two two-way nested domains centred in the Iberian Peninsula. The larger external domain's resolution was $36 \mathrm{~km}$, and the internal one's was $9 \mathrm{~km}$. Figure 1 shows the position of the two domains. The number of vertical levels is 50 .

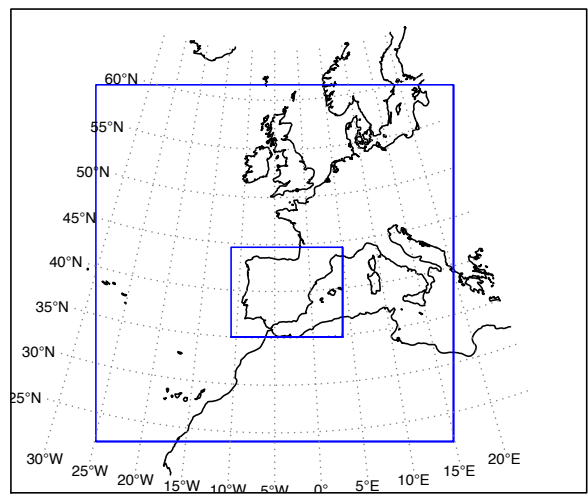

Fig. 1 Map showing the position of the two domains used in the simulation.

Every 6 hours, the boundary conditions, including SST and deep-soil temperature updates, were provided, and analysis nudging was applied in the external domain beyond the PBL. The WRF physical configuration was: Microphysics - WRF Single-Moment 6-class [21]; Longwave/Shortwave Radiation - RRTMG [22]; Surface Layer - MM5 similarity [23]; Planetary Boundary Layer - Yonsei University [24, 25]; Land Surface - Noah Land Surface Model [26]; Cumulus Parametrization - Grell-Freitas [27]. The model was run at intervals of 6 years, disregarding the first as a spin-up, and combining the results at the end. The version of WRF used was that modified by Fita et al. [28] which allows the user to obtain extreme values from the data simulated at each step of the modeling process.

In the inner domain, we focused on the region of Extremadura (Figure 2).

To compare with the results obtained with our statistical model, a set of extreme temperature observed at 28 meteorological observatories sited in the cited region of Extremadura has been used in the period 1981-2015. Both for the WRF model and the observed data, the extreme temperature is the largest temperature during the summer season (June, July and August). 


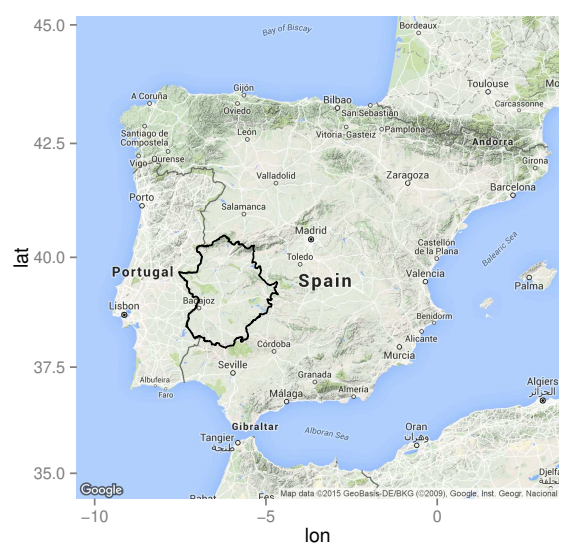

\section{Topography Extremadura}

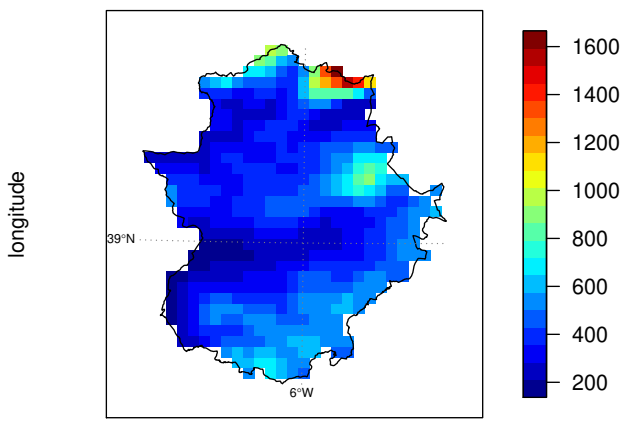

latitude

Fig. 2 Location of the Extremadura region in the Iberian Peninsula (left). Topographic map of Extremadura as seen by the WRF model (right). The scale is in metres above sea level.

\section{Statistical Model}

As was noted in the Introduction, we shall use a Bayesian hierarchical model framework in which to address the problem of estimating the parameters of a spatial Extreme Value model. The main idea behind such a hierarchical framework is that of conditional thinking. One important result of probability theory is that, for a set of statistical variables $X_{1}, X_{2}, \ldots, X_{n}$, the joint probability distribution function $\mathbf{P}\left(x_{1}, x_{2}, \ldots, x_{n}\right)$ ( $x_{i}$ being a possible value for the random variable $X_{i}$ ) can be expressed in terms of the conditional distribution as $\mathbf{P}\left(x_{1}, x_{2}, \ldots, x_{n}\right)=\mathbf{P}\left(x_{1} \mid x_{2}, x_{3}, \ldots x_{n}\right) \mathbf{P}\left(x_{2} \mid x_{3}, \ldots x_{n}\right) \cdots \mathbf{P}\left(x_{n}\right)$. It is then expected that the conditional distributions which appear on the right hand side of the above equation are easier to estimate than the distribution on the left hand side. The parameters that may appear in the statistical distributions are estimated by means of a Bayesian formalism in which both the parameters and the variables are random. A useful way to break up the whole distribution into its conditional components is that proposed in [29] (see also [30]):

Stage 1 (data model) $\mathbf{P}$ (data $\mid$ process, parameters)

Stage 2 (process model) $\mathbf{P}$ (process $\mid$ parameters)

Stage 3 (parameter model) $\mathbf{P}$ (parameters)

In the first data stage, it is supposed that the observed noisy data depend on a latent (unknown) process plus various parameters. In the second stage, a model is proposed for the latent process through a conditional probability. In the third stage, probability distributions for the parameters are introduced to take into account the uncertainties that exist about them. Bayes' theorem allows one to determine the distribution of the model's parameters once the 
data have been observed. The corresponding relationship is

$\mathbf{P}$ (process, parameters | data)

$\propto \mathbf{P}($ data $\mid$ process, parameters $) \cdot \mathbf{P}($ process $\mid$ parameters $) \cdot \mathbf{P}($ parameters $)$.

The distribution on the left hand side is termed the posterior distribution, the distribution $\mathbf{P}$ (parameters) to the far right the prior distribution, and the middle distribution $\mathbf{P}$ (data|process, parameters) the likelihood. One of the problems with Equation (1) is that the proportionality constant is unknown. Its evaluation is only feasible in simple problems. The introduction of numerical techniques such as the Markov chain Monte Carlo (MCMC) methods ([31]), however, has allowed numerical simulation of the posterior distribution of the parameters, in particular, by obtaining a sample of the parameters of interest. In the following subsections, we shall describe the proposed model in greater detail.

This hierarchical model may be placed in a more climatological context, see for example ([32], [10]). It is well known that the weather, that is the state of the atmosphere at a given time and place, may be considered as a random process and the relevant weather variables (pressure, temperature, etc) are random variables. The values of these random variables are expressed by probability distributions whose parameters may be considered as the climate of that place. This process corresponds with the Stage 1 of the hierarchical model. Furthermore the climate, represented by the parameters of the distribution, is considered a random process expressed by a probability distribution in the Stage 2. Finally, in the third Stage, a probability distribution is proposed for the prior of the parameters that appear in the Stage 2.

\subsection{First stage (data model)}

In the first stage, we suppose that the block extreme data at observatory $s$ follow a GEV distribution, where the probability distribution function can be expressed as

$\mathbf{P}\left(Y_{s} \leq y \mid \mu_{s}, \sigma_{s}, \xi_{s}\right)=\exp \left\{-\left[1+\xi_{s}\left(\frac{y-\mu_{s}}{\sigma_{s}}\right)\right]^{-1 / \xi_{s}}\right\}, \quad 1+\xi_{s}\left(\frac{y-\mu_{s}}{\sigma_{s}}\right)>0$.

In this study, the block extreme data are maximum temperatures in the summer season (June-July-August). It is also supposed that, conditional on $\mu_{s}, \sigma_{s}$ and $\xi_{s}$, the block extremes are spatially and temporally conditionally independent, i.e., the block extreme at observatory $s$ conditioned on $\mu_{s}, \sigma_{s}$, and $\xi_{s}$ is independent of the block extreme at observatory $s^{\prime}$, and that the block extreme observed at time $t$ is independent of that observed at time $t^{\prime}$. The scale parameter $\sigma_{s}$ represents the spread of the distribution, with a greater value representing greater dispersion of the maximum temperature. 
The shape parameter $\xi_{s}$ represents the tail behaviour of the extreme distribution - a negative parameter corresponds to a bounded tail, otherwise the tail is unbounded.

\subsection{Second stage (process model)}

In the second stage, the parameters of the GEV distribution are assumed to follow a spatio-temporal model, i.e., to depend on spatial coordinates $\mathbf{s}$ and time $t$. At this point, it is important to distinguish between stationary and non-stationary models. In the former, the GEV parameters do not depend on time, while in the latter they do. In the stationary case, the parameters of the GEV distributions are assumed to take the form

$$
\begin{aligned}
& \mu(\mathbf{s})=X_{\mu}(\mathbf{s}) \cdot \boldsymbol{\alpha}_{\mu}+W_{\mu}(\mathbf{s})+\epsilon_{\mu} \\
& \sigma(\mathbf{s})=X_{\sigma}(\mathbf{s}) \cdot \boldsymbol{\alpha}_{\sigma}+W_{\sigma}(\mathbf{s})+\epsilon_{\sigma} \\
& \xi(\mathbf{s})=X_{\xi}(\mathbf{s}) \cdot \boldsymbol{\alpha}_{\xi}+W_{\xi}(\mathbf{s})+\epsilon_{\xi}
\end{aligned}
$$

where $X_{\mu, \sigma, \xi}(\mathbf{s})$ are $p$ spatial covariates (geographical coordinates), that may be different for each parameter; $\boldsymbol{\alpha}_{\mu, \sigma, \xi}$ is a set of $p$ regression parameters, $W_{\mu, \sigma, \xi}(\mathbf{s})$ are spatial models capturing the associations between different sites (grid cells), and $\epsilon_{\mu, \sigma, \xi}$ represent noise unaccounted for in the spatial models (in the spatial data community, generally known as the nugget effect), and $\mathbf{s}$ denotes a spatial coordinate. In the non-stationary case, a linear time dependence is included in the location parameter:

$$
\mu(\mathbf{s}, t)=X_{\mu}(\mathbf{s}) \cdot \boldsymbol{\alpha}_{\mu}+W_{\mu}(\mathbf{s})+\left(X_{\mu}^{\prime}(\mathbf{s}) \cdot \gamma_{\mu}+W_{\mu}^{\prime}+\epsilon_{\mu}^{\prime}\right)\left(t-t_{0}\right)+\epsilon_{\mu} .
$$

This equation can be rewritten as

$$
\mu(\mathbf{s}, t)=\mu_{0}(\mathbf{s})+\mu_{1}(\mathbf{s})\left(t-t_{0}\right) .
$$

In Equation (6), it has been assumed that the temporal-trend coefficient may depend on geographical factors through the covariates $X_{\mu}^{\prime}$, and that nearer sites could show a stronger temporal relationship through the spatial model $W_{\mu}^{\prime}$. The spatial models $W_{\kappa}(\mathbf{s}), \kappa=\mu, \sigma, \xi ; W_{\mu}^{\prime}(\mathbf{s})$ are assumed to be of the form

$$
W_{\kappa}(\mathbf{s})=Z \Psi_{\kappa}\left(\mathbf{s}^{\prime}\right) ; \quad \kappa=\mu, \sigma, \xi
$$

where $Z$ is an $n \times r$ matrix and $\Psi_{\kappa}\left(\mathbf{s}^{\prime}\right)$ is a spatial process of dimension $r$. This spatial process is modeled as a multivariate Gaussian process with zero mean and covariance matrix $\boldsymbol{\Sigma}$, i.e.,

$$
\Psi_{\kappa}\left(\mathbf{s}^{\prime}\right) \sim M V N\left(\mathbf{0} ; \boldsymbol{\Sigma}_{\kappa}\right) ; \quad \kappa=\mu, \sigma, \xi
$$


with the symbol $\sim$ we means 'distributed as'. The $r \times r$ covariance matrix defines the covariance among the $r$ spatial locations, and is assumed to be of the form

$$
\left.\boldsymbol{\Sigma}_{\kappa}\left(s^{\prime}, s^{\prime}\right)=\beta_{\kappa 1}^{2} \exp \left(-\left\|s_{i}^{\prime}-s_{j}^{\prime}\right\|\right) / \beta_{\kappa 2}\right)=\beta_{\kappa 1}^{2} R_{\kappa}\left(\beta_{\kappa 2}\right)
$$

where $R_{\kappa}$ is the correlation matrix. The parameter $\beta_{\kappa 2}$ (also called the range parameter) defines the strength of the association in the relationships among the spatial sites. The variance parameter $\beta_{\kappa 1}^{2}$ is also known as the sill in the geostatistics community. The residual noise $\epsilon_{\kappa}$ is modeled as a Gaussian process of zero mean and variance $\tau_{\kappa}^{2}$ which is assumed to be constant everywhere.

The reason for the introduction into our model of a matrix $Z$ that transforms an r-spatial process into an n-spatial process is the following. The number of grid cells increases enormously as the resolution of the model increases. For example, for our region of Extremadura and with a WRF resolution of $9 \mathrm{~km}$, there are 957 grid cells. Such a large number of cells makes the numerical problem nearly unfeasible (the covariance matrix would be $957 \times 957$ and we would have to invert such a large matrix at each MCMC step). To lighten the numerical burden, we followed the method proposed by [33], and modified by [34] (see also [35]). Those authors proposed replacing the $W_{\kappa}$ process by what they called a predictive process $\tilde{W}_{\kappa}$, defined as

$$
\tilde{W}_{\kappa}(s)=E\left(W_{\kappa}(s) \mid \Psi_{\kappa}\left(s^{\prime}\right)\right)=\boldsymbol{\Sigma}_{\kappa}\left(s, s^{\prime}\right) \cdot \boldsymbol{\Sigma}_{\kappa}^{-1}\left(s^{\prime}, s^{\prime}\right) \Psi_{\kappa}\left(s^{\prime}\right)=Z_{\kappa} \Psi_{\kappa}\left(s^{\prime}\right),
$$

where $\boldsymbol{\Sigma}_{\kappa}\left(s, s^{\prime}\right)$ is the $n \times r$ covariance matrix between an s-site and an s'site. With a value $r \ll n$, the problem of inverting an $n \times n$ matrix at each MCMC step reduces to that of inverting an $r \times r$ matrix. In this work, we have subsampling the WRF grid every three rows and columns which give a r-matrix of $110 \times 110$ elements. The substitution of the $W_{\kappa}$ process by the predictive $\tilde{W}_{\kappa}$ process has the consequence of reducing the variance of the spatial process. To compensate for this, [34] proposes increasing the nugget variance $\tau^{2} I$ by the amount

$$
\operatorname{Diagonal}\left(I-\boldsymbol{\Sigma}_{\kappa}\left(s, s^{\prime}\right) \boldsymbol{\Sigma}_{\kappa}^{-1}\left(s^{\prime}, s^{\prime}\right) \boldsymbol{\Sigma}_{\kappa}\left(s^{\prime}, s\right)\right) .
$$

To close this second stage, Equation (3) may be expressed as normal distributions: for the stationary model,

$$
\begin{aligned}
& \mu(\mathbf{s}) \sim N\left(\mathbf{X}_{\mu}(\mathbf{s}) \cdot \boldsymbol{\alpha}_{\mu}+W_{\mu}(\mathbf{s}), \hat{\tau}_{\mu}^{2}\right) \\
& \sigma(\mathbf{s}) \sim N\left(\mathbf{X}_{\sigma}(\mathbf{s}) \cdot \boldsymbol{\alpha}_{\sigma}+W_{\sigma}(\mathbf{s}), \hat{\tau}_{\sigma}^{2}\right) \\
& \xi(\mathbf{s}) \sim N\left(\mathbf{X}_{\xi}(\mathbf{s}) \cdot \boldsymbol{\alpha}_{\xi}+W_{\xi}(\mathbf{s}), \hat{\tau}_{\xi}^{2}\right),
\end{aligned}
$$

and for the non-stationary model,

$$
\mu_{1}(\mathbf{s}) \sim N\left(\mathbf{X}_{\mu}(\mathbf{s}) \cdot \boldsymbol{\alpha}_{\mu}+W_{\mu}(\mathbf{s}), \hat{\tau}_{\mu}^{2}\right)
$$




$$
\begin{aligned}
\mu_{2}(\mathbf{s}) & \sim N\left(\mathbf{X}_{\mu}^{\prime}(\mathbf{s}) \cdot \boldsymbol{\gamma}_{\mu}+W_{\mu}^{\prime}(\mathbf{s}), \hat{\tau}_{\mu}^{\prime 2}\right) \\
\sigma(\mathbf{s}) & \sim N\left(\mathbf{X}_{\sigma}(\mathbf{s}) \cdot \boldsymbol{\alpha}_{\sigma}+W_{\sigma}(\mathbf{s}), \hat{\tau}_{\sigma}^{2}\right) \\
\xi(\mathbf{s}) & \sim N\left(\mathbf{X}_{\xi}(\mathbf{s}) \cdot \boldsymbol{\alpha}_{\xi}+W_{\xi}(\mathbf{s}), \hat{\tau}_{\xi}^{2}\right),
\end{aligned}
$$

where the $\hat{\tau}_{i}$ represent the increased nugget variances.

\subsection{Third stage (prior model)}

In the third stage, prior distributions have to be provided for the parameters used in the previous two stages, in particular, for the spatial regression coefficients $\boldsymbol{\alpha}_{\kappa}$, the parameters of the covariance model $\beta_{\kappa 1}^{2}, \beta_{\kappa 2}$, the nugget variances $\tau_{\kappa}^{2}$, and, in the non-stationary case, the trend parameters $\gamma_{\kappa}, \beta_{\kappa 1}^{\prime 2}, \beta_{\kappa 2}^{\prime}, \tau_{\kappa}^{\prime 2}$. As the present study area is not too large, we took as covariates $X$ the grid cell heights provided by the WRF topography (see Figure 2, right). Therefore, the term $X(\mathbf{s}) \cdot \boldsymbol{\alpha}_{\kappa}$ can be written as $\alpha_{\kappa 0}+h_{s} \alpha_{\kappa 1}$, so that we need to provide priors for $\alpha_{\kappa 0}, \alpha_{\kappa 1}$. Gaussian distributions were used for both cases.

$$
\alpha_{\kappa j} \sim N\left(a_{\alpha_{\kappa j}}, b_{\alpha_{\kappa j}}^{2}\right), \quad \kappa=\{\mu, \sigma, \xi\}, j=\{0,1\},
$$

where the hyperparameters of mean $\left(a_{\alpha_{\kappa j}}\right)$ and variance $\left(b_{\alpha_{\kappa j}}^{2}\right)$ were chosen appropriately in such a way that the distribution was either non- or only weakly informative with no extra information about the parameters, e.g., $\alpha_{\mu j} \sim N(0,10000)$. A similar model (Gaussian) was selected for the trend parameter $\gamma$ in the non-stationary model. The sill parameters $\beta_{\kappa 1}^{2}$ and the nugget variances $\tau_{\kappa}^{2}$ were parametrized by inverse gamma distributions. A uniform distribution was chosen for the range $\beta_{\kappa 2}$.

\subsection{Parameter estimation}

Pulling together the different parts of the hierarchical model, and taking into account Bayes' theorem as expressed in Equation (1), for the stationary model, the posterior distribution of the parameters is given by the expression

$$
\begin{aligned}
& \mathbf{P}\left(\boldsymbol{\mu}, \boldsymbol{\sigma}, \boldsymbol{\xi}, \mathbf{W}_{\mu}, \mathbf{W}_{\sigma}, \mathbf{W}_{\xi}, \boldsymbol{\alpha}_{\mu}, \boldsymbol{\alpha}_{\sigma}, \boldsymbol{\alpha}_{\xi}, \boldsymbol{\beta}_{\mu}, \boldsymbol{\beta}_{\sigma}, \boldsymbol{\beta}_{\xi}, \tau_{\mu}^{2}, \tau_{\sigma}^{2}, \tau_{\xi}^{2} \mid \boldsymbol{Y}, \boldsymbol{X}\right) \\
& \propto \prod_{s=1}^{n g r i d} \prod_{t=1}^{n d a t} \mathbf{G E V}\left(y_{t s} \mid \boldsymbol{\mu}, \boldsymbol{\sigma}, \boldsymbol{\xi}\right) \\
& \text { - } \mathbf{P}\left(\boldsymbol{\mu} \mid \boldsymbol{X}_{\mu}, \boldsymbol{\alpha}_{\mu}, W_{\mu}, \tau_{\mu}^{2}\right) \mathbf{P}\left(\boldsymbol{\sigma} \mid \boldsymbol{X}_{\sigma}, \boldsymbol{\alpha}_{\sigma}, W_{\sigma}, \tau_{\sigma}^{2}\right) \mathbf{P}\left(\boldsymbol{\xi} \mid \boldsymbol{X}_{\xi}, \boldsymbol{\alpha}_{\xi}, W_{\xi}, \tau_{\xi}^{2}\right) \\
& \text { - } \mathbf{P}\left(\boldsymbol{\Psi}_{\mu} \mid \boldsymbol{\beta}_{\mu}\right) \mathbf{P}\left(\boldsymbol{\Psi}_{\sigma} \mid \boldsymbol{\beta}_{\sigma}\right) \mathbf{P}\left(\boldsymbol{\Psi}_{\xi} \mid \boldsymbol{\beta}_{\xi}\right) \\
& \text { - } \mathbf{P}\left(\alpha_{\mu 0}\right) \mathbf{P}\left(\alpha_{\mu 1}\right) \mathbf{P}\left(\alpha_{\sigma 0}\right) \mathbf{P}\left(\alpha_{\sigma 1}\right) \mathbf{P}\left(\alpha_{\xi 0}\right) \mathbf{P}\left(\alpha_{\xi 1}\right) \\
& \text { - } \mathbf{P}\left(\beta_{\mu 0}^{2}\right) \mathbf{P}\left(\beta_{\mu 1}\right) \mathbf{P}\left(\beta_{\sigma 0}^{2}\right) \mathbf{P}\left(\beta_{\sigma 1}\right) \mathbf{P}\left(\beta_{\xi 0}^{2}\right) \mathbf{P}\left(\beta_{\xi 1}\right) \mathbf{P}\left(\tau_{\mu}^{2}\right) \mathbf{P}\left(\tau_{\sigma}^{2}\right) \mathbf{P}\left(\tau_{\xi}^{2}\right),
\end{aligned}
$$

where $W_{\kappa}, \kappa=\mu, \sigma, \xi$ has been estimated by its definition $W_{\kappa}=Z \Psi_{\kappa}$. For the non-stationary model, the posterior distribution is given by the expression

$$
\mathbf{P}\left(\boldsymbol{\mu}, \boldsymbol{\sigma}, \boldsymbol{\xi}, \mathbf{W}_{\mu}, \mathbf{W}^{\prime}{ }_{\mu}, \mathbf{W}_{\sigma}, \mathbf{W}_{\xi}, \boldsymbol{\alpha}_{\mu}, \boldsymbol{\gamma}_{\mu}, \boldsymbol{\alpha}_{\sigma}, \boldsymbol{\alpha}_{\xi}, \boldsymbol{\beta}_{\mu}, \boldsymbol{\beta}_{\mu}^{\prime} \boldsymbol{\beta}_{\sigma}, \boldsymbol{\beta}_{\xi}, \tau_{\mu}^{2}, \tau_{\mu}^{\prime 2}, \tau_{\sigma}^{2}, \tau_{\xi}^{2} \mid \boldsymbol{Y}, \boldsymbol{X}, f\right.
$$




$$
\begin{aligned}
& \propto \prod_{s=1}^{n g r i d} \prod_{t=1}^{n d a t} \operatorname{GEV}\left(y_{t s} \mid \boldsymbol{\mu}, \boldsymbol{\sigma}, \boldsymbol{\xi}, f(t)\right) \\
& \text { - } \mathbf{P}\left(\boldsymbol{\mu}_{\mathbf{1}} \mid \boldsymbol{X}_{\boldsymbol{\mu}}, \boldsymbol{\alpha}_{\mu}, W_{\mu}, \tau_{\mu}^{2}\right) \mathbf{P}\left(\boldsymbol{\mu}_{\mathbf{2}} \mid \boldsymbol{X}_{\boldsymbol{\mu}}^{\prime}, \boldsymbol{\gamma}_{\mu}, W_{\mu}^{\prime}, \tau_{\mu}^{\prime 2}\right) \\
& \text { - } \mathbf{P}\left(\boldsymbol{\sigma} \mid \boldsymbol{X}_{\boldsymbol{\sigma}}, \boldsymbol{\alpha}_{\sigma}, W_{\sigma}, \tau_{\sigma}^{2}\right) \mathbf{P}\left(\boldsymbol{\xi} \mid \boldsymbol{X}_{\boldsymbol{\xi}}, \boldsymbol{\alpha}_{\xi}, W_{\xi}, \tau_{\xi}^{2}\right) \\
& \text { - } \mathbf{P}\left(\boldsymbol{\Psi}_{\mu} \mid \boldsymbol{\beta}_{\mu}\right) \mathbf{P}\left(\boldsymbol{\Psi}_{\mu}^{\prime} \mid \beta^{\prime}{ }_{\mu}\right) \\
& \text { - } \mathbf{P}\left(\boldsymbol{\Psi}_{\sigma} \mid \boldsymbol{\beta}_{\sigma}\right) \mathbf{P}\left(\boldsymbol{\Psi}_{\xi} \mid \boldsymbol{\beta}_{\xi}\right) \\
& \text { - } \mathbf{P}\left(\alpha_{\mu 0}\right) \mathbf{P}\left(\alpha_{\mu 1}\right) \mathbf{P}\left(\gamma_{\mu 0}\right) \mathbf{P}\left(\gamma_{\mu 1}\right) \mathbf{P}\left(\alpha_{\sigma 0}\right) \mathbf{P}\left(\alpha_{\sigma 1}\right) \mathbf{P}\left(\alpha_{\xi 0}\right) \mathbf{P}\left(\alpha_{\xi 1}\right) \\
& \text { - } \mathbf{P}\left(\beta_{\mu 0}^{2}\right) \mathbf{P}\left(\beta_{\mu 1}\right) \mathbf{P}\left({\beta_{\mu 0}^{\prime}}^{2}\right) \mathbf{P}\left(\beta_{\mu 1}^{\prime}\right) \mathbf{P} p\left(\beta_{\sigma 0}^{2}\right) \mathbf{P}\left(\beta_{\sigma 1}\right) \mathbf{P}\left(\beta_{\xi 0}^{2}\right) \mathbf{P}\left(\beta_{\xi 1}\right) \\
& \text { - } \mathbf{P}\left(\tau_{\mu}^{2}\right) \mathbf{P}\left(\tau_{\mu}^{\prime 2}\right) \mathbf{P}\left(\tau_{\sigma}^{2}\right) \mathbf{P}\left(\tau_{\xi}^{2}\right),
\end{aligned}
$$

where $\boldsymbol{\mu}=\boldsymbol{\mu}_{0}+f(t) \boldsymbol{\mu}_{1}$, with $f(t)=\left(t-t_{0}\right)$ being the linear temporal-trend function. The simulation of the posterior distribution was carried out by means of an MCMC method, in particular using a Gibbs sampler with embedded Metropolis-Hastings steps (see [31] for more details about MCMC methods). The MCMC was iterated for 50000 samples with a burn-in period of 30000 samples to allow the MCMC to reach the stationary state. To avoid the autocorrelation that appears in the MCMC, we kept one out of ten samples for the subsequent calculations. To test the convergence of the chains, four chains were constructed starting with different values of the parameters being simulated. The Gelman-Rubin diagnostic convergence test (see [36]) was used to evaluate the convergence of these four chains. In most cases, that burn-in period was sufficient for convergence to be reached.

The code used to carry out the simulations was written in FORTRAN, following quite closely the procedure in [37]. For the Gelman-Rubin diagnostic test, the CODA package of the $\mathrm{R}$ language was used, and the maps and figures were prepared using the $\mathrm{R}$ packages fields and $s p$.

\subsection{Checking the models}

An important step in a statistical analysis is to assess whether the observed data are indeed fitted by the proposed statistical model or models. A two step procedure has been followed. In the first step, the models were ranked according to a model comparison tool such as the WAIC (Widely Applicable Information Criterion) [38]. In the second step, the chosen model was assessed versus the data by means of a Bayesian p-value procedure. In both steps the posterior predictive distribution has been used. The posterior predictive distribution (PPD) is defined as [40]

$$
\mathbf{P}\left(y^{\mathrm{rep}} \mid M, y\right)=\int \mathbf{P}\left(y^{\mathrm{rep}} \mid M, \theta\right) \mathbf{P}(\theta \mid M, y) d \theta,
$$

which gives the probability of obtaining new replicated data given the model $M$ and the observed data $y$. In this equation, $\theta$ represents the parameters of the model, $\mathbf{P}(\theta \mid M, y)$ the posterior distribution, and $\mathbf{P}\left(y^{\mathrm{rep}} \mid M, \theta\right)$ the likelihood. 
The WAIC criterion to rank the models is defined by the equation

$$
\mathrm{WAIC}=-2 \log \left(\prod_{i=1}^{n} \mathbf{P}\left(y_{i}\right)\right)+p_{\text {waic }}=-2 \sum_{i=1}^{n} \log \left(\mathbf{P}\left(y_{i}\right)\right)+p_{\text {waic }},
$$

where $\mathbf{P}\left(y_{i}\right)$ is the PPD given by Equation (14), and $p_{\text {waic }}$ is a term that measures both the bias introduced into the test from using the data twice once to estimate the parameters, and a second time to use them in the test (see [38]) - and the complexity of the model (penalizing models with more parameters). The term $-2 \sum_{i=1}^{n} \log \left(\mathbf{P}\left(y_{i}\right)\right)$ is estimated by the equation

$$
-2 \sum_{i=1}^{n} \log \left(\mathbf{P}\left(y_{i}\right)\right) \approx-2 \sum_{i=1}^{n} \log \left[\frac{1}{J} \sum_{j=1}^{J}\left(\mathbf{P}\left(y_{i} \mid \theta^{j}\right)\right)\right]
$$

where $J$ is the number of samples taken from the MCMC method and $\theta^{j}$ are the estimated parameters of the model. The $p_{\text {waic }}$ parameter is defined by the expression

$$
p_{\text {waic }}=\sum_{i=1}^{n} \operatorname{var}_{\text {post }}\left[\log \left(\mathbf{P}\left(y_{i}\right)\right)\right]
$$

where $\operatorname{var}_{\text {post }}$ is the operator variance and is estimated as

$$
\operatorname{var}_{\text {post }}(x)=\frac{1}{J-1} \sum_{j=1}^{J}\left(x_{j}-\bar{x}\right) ; \quad x_{j}=\log \left(\mathbf{P}\left(y_{i} \mid \theta^{j}\right)\right) .
$$

As before, $J$ is the number of samples taken from the MCMC. The choice of the WAIC criterion is motivated by the fact that it is easy to calculate from the MCMC and that it has the property of being asymptotically equivalent to a leave-one-out cross-validation (LOO-CV) test (see [38]).

In the second step, the Bayesian p-value has been evaluated as follows. Let $T(y)$ be some feature of the data we are interested in, for example, the skewness of the data, and we want to see whether the model predicts this feature reasonably well. A measure of the agreement is the Bayesian p-value defined by [39]

$$
p_{b}(y)=\mathbf{P}\left(T\left(y^{\mathrm{rep}}\right) \geq T(y) \mid M, y\right) .
$$

A $p_{b}$-value that is too small or too large would indicate that the model does not reproduce the data (or at least some features of the data) well. One way to calculate the $p_{b}$-value is from the MCMC. As was noted above, an MCMC method allows one to obtain values of the parameter $\theta$ from the posterior distribution $\mathbf{P}(\theta \mid y)$. For each simulated $\theta$ value, a $y^{\text {rep }}$ value is simulated by using the posited model, and then the statistic $T\left(y^{\mathrm{rep}}\right)$ is computed. Evaluating the number of times that $T\left(y^{\text {rep }}\right) \geq T(y)$ gives the $p_{b}$-value as 


$$
p_{b}=\frac{\#\left(T\left(y^{\mathrm{rep}}\right) \geq T(y)\right)}{J},
$$

with $J$ being the number of samples of $\theta$ taken from the Markov chain.

\subsection{Prediction at a non-grid site}

Once we have fitted the model, we are in a position to predict variables of interest at a non-grid site. In particular, we are interested in predicting the parameters of the GEV distribution $\boldsymbol{\mu}, \boldsymbol{\sigma}, \boldsymbol{\xi}$ in order to estimate, for example, the $T$-year return levels at those non-grid sites. We shall illustrate the method developed for the location parameter $\boldsymbol{\mu}$. Based on Equation (3), we shall assume that if $\mu_{0}$ is the location parameter at a non-grid site then the joint statistical distribution of the location parameter at the grid and non-grid sites is given by

$$
\left(\begin{array}{c}
\mu_{0} \\
\boldsymbol{\mu}
\end{array}\right) \sim \operatorname{MVN}\left(\begin{array}{c|cc}
m_{0} & \sigma_{0} & \boldsymbol{\Sigma}_{0 n} \\
\mathbf{m} & \boldsymbol{\Sigma}_{n 0} & \boldsymbol{\Sigma}_{n n}
\end{array}\right)
$$

where MVN represents the multivariate normal distribution, $m_{0}, \mathbf{m}$ are the means of the distributions at the non-grid site and grid sites respectively, $\sigma_{0}$ is the variance at the non-grid site, $\boldsymbol{\Sigma}_{n n}$ is the $n \times n$ covariance matrix at the grid sites, and $\boldsymbol{\Sigma}_{0 n}, \boldsymbol{\Sigma}_{n 0}$ are the $1 \times n, n \times 1$ covariance matrices between the non-grid and grid sites. A result from multinormal distribution theory allows us to say that the conditional distribution of $\mu_{0}$ given $\boldsymbol{\mu}$, i.e. $P\left(\mu_{0} \mid \boldsymbol{\mu}\right)$, is normal with mean given by

$$
m_{1}=m_{0}+\boldsymbol{\Sigma}_{0 n} \boldsymbol{\Sigma}_{n n}^{-1}(\boldsymbol{\mu}-\mathbf{m})
$$

and variance

$$
\sigma_{1}=\sigma_{0}-\boldsymbol{\Sigma}_{0 n} \boldsymbol{\Sigma}_{n n}^{-1} \boldsymbol{\Sigma}_{n 0} .
$$

Once the mean $m_{1}$ and variance $\sigma_{1}$ have been estimated, one can take samples of the location parameter at the non-grid site. According to Equations (3) to (5), the $\boldsymbol{\mu}$ distribution is

$$
\boldsymbol{\mu} \sim \operatorname{MVN}\left(X \cdot \boldsymbol{\alpha}_{u}+W_{\mu}, \tau_{\mu}^{2} \mathbf{I}\right)
$$

where $W_{\mu}$ is the spatial process given by $W_{\mu}=Z \Psi_{\mu}$, with $Z$ being an $n \times r$ matrix and $\Psi_{\mu}$ is an $r \times r$ MVN process with zero mean and variance $\boldsymbol{\Sigma}_{\mu}$ (Equations (8) and (9)). Integrating in $\Psi_{\mu}$, the above distribution may be expressed as

$$
\boldsymbol{\mu} \sim \operatorname{MVN}\left(X \cdot \boldsymbol{\alpha}_{\mu}, Z \boldsymbol{\Sigma}_{\mu} Z^{T}+\tau_{\mu}^{2} \mathbf{I}\right) .
$$

From this expression, one can take $\mathbf{m}=X \cdot \boldsymbol{\alpha}_{\mu}$ and $m_{0}=X_{0} \cdot \boldsymbol{\alpha}_{\mu}$. Taking into account expression (10), the $r \times r$ covariance matrix $\boldsymbol{\Sigma}_{\mu}$ is given by

$$
\left.\boldsymbol{\Sigma}_{\mu}\left(\mathbf{s}^{\prime}, \mathbf{s}^{\prime}\right)=\beta_{\mu 1}^{2} \exp \left(-\left\|\mathbf{s}_{i}^{\prime}-\mathbf{s}_{j}^{\prime}\right\|\right) / \beta_{\mu 2}\right), \quad i, j=1, \cdots, r .
$$

The $Z$ matrix is given by (see Equation (11)): 


$$
Z=\boldsymbol{\Sigma}_{\mu}\left(\mathbf{s}, \mathbf{s}^{\prime}\right) \cdot \boldsymbol{\Sigma}_{\mu}^{-1}\left(\mathbf{s}^{\prime}, \mathbf{s}^{\prime}\right) .
$$

Lastly, the covariance between the non-grid and a grid site is taken as

$$
\left.\boldsymbol{\Sigma}_{n 0}=\beta_{\mu 1}^{2} \exp \left(-\mathbf{s}_{i}-\mathbf{s}_{0}\right) / \beta_{\mu 2}\right),
$$

and $\sigma_{0}=\tau_{\mu}^{2}$.

\section{Results}

Before entering to describe the results given by the statistical model we would like to know if the climate of the region (represented by the summer maximum temperature) is well described by the WRF model. Figure 3 shows a map of the mean of summer extreme temperature for the study period (1981-2015) given by the Stead data base ([41]) (left) and by the WRF model (right). As it may be seen the spatial distribution of the maximum temperature are quite similar in both maps so that we have relative confidence in the results given by the WRF model.

Mean of TMax summer (Stead)

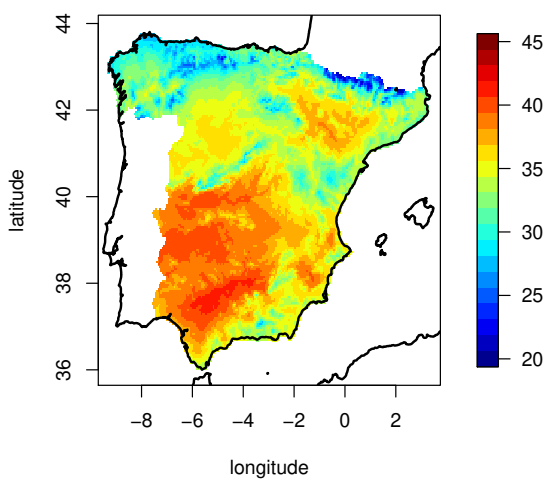

Mean of Tmax summer (WRF)

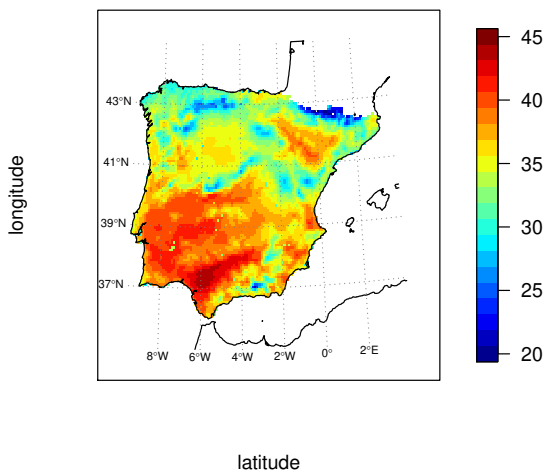

Fig. 3 Mean of the summer extreme temperature during the period 1981-2015 given by the WRF model (right) for the Iberian Peninsula and during the period 1981-2014 given by Stead database for Spain.

Moving into the statistical model, it is firstly necessary to establish the external covariate term $X$. Because the spatial domain is not too large, and given the influence of altitude on temperature, we chose the height of the grid cell to be our covariate. Prior to their introduction into the model, the heights were normalized as

$$
\tilde{h}_{s}=\frac{h_{s}^{\text {grid }}-\min _{s}\left\{h_{s}\right\}}{\max _{s}\left\{h_{s}\right\}-\min _{s}\left\{h_{s}\right\}}
$$


where $h_{s}^{\text {grid }}$ is the altitude (height above mean sea level) of grid cell $s$, and $\max _{s}\left\{h_{s}\right\}$ and $\min _{s}\left\{h_{s}\right\}$ are the maximum and minimum grid cell altitudes, respectively, so that the new height covariate $\tilde{h}_{s}$ is 0 for the lowest grid cell and 1 for the highest. With this choice of covariate, the spatial-trend term in the location parameter $\boldsymbol{\mu}$ (stationary models) and $\boldsymbol{\mu}_{0}$ (non-stationary models) is

$$
\mathbf{X}_{\mu}(s) \cdot \boldsymbol{\alpha}_{\mu}=\alpha_{0}+\tilde{h}_{s} \alpha_{1},
$$

and in the temporal-trend coefficient $\boldsymbol{\mu}_{1}$ is

$$
\mathbf{X}_{\mu}^{\prime}(s) \cdot \gamma_{\mu}=\gamma_{0}+\tilde{h}_{s} \gamma_{1} .
$$

The linear temporal-trend function $f(t)=t-t_{0}$ was also normalized to the interval $(-1,1)$ by the expression

$$
\hat{f}(t)=\frac{2}{t_{1}-t_{0}}\left(t-t_{0}\right)-1,
$$

with $t_{0}, t_{1}$ being the first and last years, respectively.

A preliminary study of the spatial models revealed that the spatial-trend coefficient $\boldsymbol{\alpha}_{\xi}$ for the shape parameter $\xi$ is not significantly different from zero. For this reason, and given that the shape parameter is the most difficult to estimate $([42],[43])$ we shall assume this shape parameter to be constant throughout the domain.

We posited various models depending on whether or not there exists a temporal trend in the location parameter, and, within them, whether there exists a dependence on height in both the location and the scale parameters. As aforementioned, to rank the proposed models, the WAIC criterion has been used. The results are shown in Table 1.

Observing column $p_{\text {waic }}$, it is seen that as the complexity of the models increases so does the complexity parameter. The results in the last column lead to non-stationary models fitting better than stationary models, with model 2110 performing the best. Therefore, this model was chosen for the next step, the assessment of the model against the data using the Bayesian p-value.

As noted above, to apply the Bayesian p-value we need to choose a statistic $T(y)$ of the data that can help determine which model best explains some feature of the data. Because we are considering a time dependence in the models, we took the Mann-Kendall test as the feature to explain. This is a non-parametric test used to determine whether a uniform trend exists in a set of independent data. The test statistic is given by the expression ([44])

$$
T(y)=\frac{1}{\sqrt{n(n-1)(2 n+5) / 18}}\left(\sum_{i=1}^{n-1} \sum_{j=i+1}^{n} \operatorname{sgn}\left(y_{j}-y_{i}\right)-\operatorname{sgn}(T)\right),
$$

where $\operatorname{sgn}(x)$ is the sign function defined as +1 if $x>0,-1$ if $x<0$, and 0 if $x=0$, and $n$ is the number of data for each grid cell. By evaluating this statistic for the 'observed' and the replicated data, we calculated the $p_{b}$-value. 
To simulate replicated data according to the PPD given by expression (14), an instance of the $\mu, \sigma, \xi$ parameters is taken from the MCMC chain, and a sample of $n$ elements is extracted using the GEV distribution. The statistic $T\left(y^{\mathrm{rep}}\right)$ is then evaluated from this sample. This procedure is repeated for each element of the chain, obtaining a sample of $J$ replicated Mann-Kendall statistics. This statistic is also evaluated for the observed data. From the replicated sample and the observed datum, the $p_{b}$ value is calculated as the percentile of the observed $T\left(y^{\text {obs }}\right)$ in the $J$ replicated Mann-Kendall statistics. Values of $p_{b}$ that are too large or too small mean that the replicated data do not explain that feature of the observed data well. If there are $N$ grid cell elements in the domain, we obtain $N p_{b}$ values. From this set, we calculated, by way of synthesis, the minimum, the 25, 50 and 75 percentiles together with the maximum. For the chosen 2110 model these values are: $0.1870,0.4080,0.4470$, $0.5440,0.9500$, respectively. Values of the 25,50 and 75 percentiles are near 0.5 , which means that for most sites the 2110 model explains quite well the temporal trend observed in the results given by the RCM.

\begin{tabular}{c|c|cc} 
Kind & Model & $p_{\text {waic }}$ & WAIC \\
\hline \hline Stationary & 200 & 816.7 & 232663 \\
& 210 & 923.8 & 231042 \\
& 220 & 923.4 & 231038 \\
\hline Non-stationary & 2000 & 819.9 & 231314 \\
& 2010 & 1051.2 & 230790 \\
& 2020 & 1050.1 & 230782 \\
& 2110 & 1079.4 & 230480 \\
& 2220 & 1079.1 & 230486 \\
\hline
\end{tabular}

Table 1 Summary of the values of the WAIC information criterion. The column headed 'Model' lists the tags used to distinguish the spatial-trend parameters used in each statistical model. These tags are: 2, dependence on the observatory's altitude; 1 , no dependence on altitude; and 0 , the parameter is considered constant throughout the domain. For the stationary models, the three parameters are location, scale, and shape, respectively. For the non-stationary models, the four parameters are location $\boldsymbol{\mu}_{0}, \boldsymbol{\mu}_{1}$, and scale and shape. Column $p_{\text {waic }}$ lists the model complexity parameter, and column WAIC, the WAIC parameter.

Let us now consider this model's results in greater detail. Firstly, Figure 4 shows the spatial regression coefficients $\alpha_{0}, \alpha_{1}$. The right-hand panel shows that the height-trend coefficient is negative with a mean value of $-15.1^{\circ} \mathrm{C}$. Therefore, given that the heights were normalized (see (29)), and considering that the maximum altitude in the model is $1794.8 \mathrm{~m}$ and the minimum is $134.8 \mathrm{~m}$, one has the result that $\alpha_{1}=-9.35^{\circ} \mathrm{C} / \mathrm{km}$, which is quite close to the theoretical adiabatic coefficient of $-9.8^{\circ} \mathrm{C} / \mathrm{km}$. It is important to bear in mind that the data correspond to summer when the temperatures are the highest and the atmospheric boundary layer is in nearly adiabatic equilibrium. This result lends added confidence to the proposed model.

Secondly, we shall show the results for the temporal-trend coefficients $\gamma_{0}, \gamma_{1}$. Although the chosen model only has the $\gamma_{0}$ coefficient, an analysis of that model with $\gamma_{1}$ present showed that coefficient to not differ from zero, i.e., 

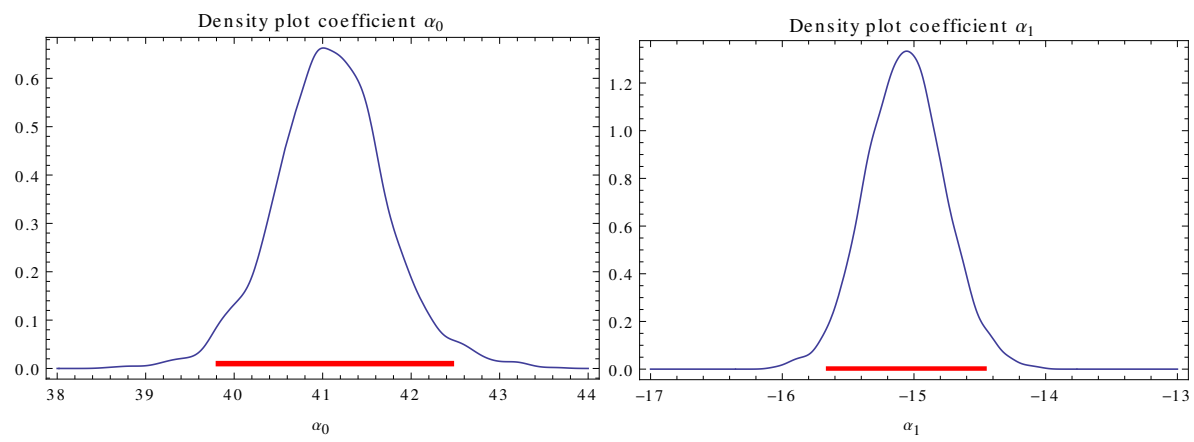

Fig. 4 Spatial-regression coefficients $\alpha_{0}$ (left) and $\alpha_{1}$ (right). The red line shows the interval $(5 \%, 95 \%)$.

the temporal-trend coefficient has no height dependence. Figure 5 shows a density plot of $\gamma_{0}$. The mean $(5 \%, 95 \%)$ is $-0.26(-0.39,-0.12)$. Taking into account

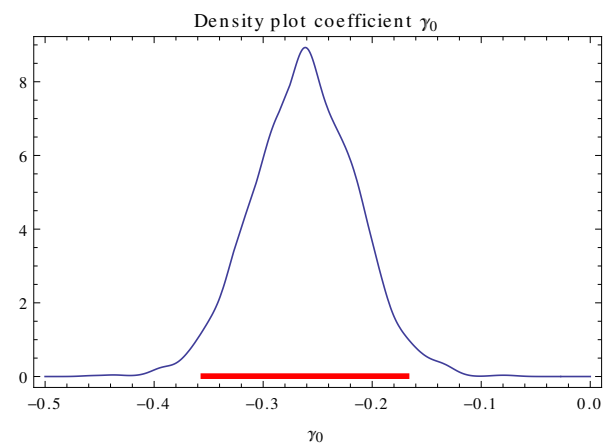

Fig. 5 Temporal-trend coefficients $\gamma_{0}$. The red line shows the interval $(5 \%, 95 \%)$.

Equation (32), one finds a temporal trend of $-0.15^{\circ} \mathrm{C}$ per decade with a $5 \%-95 \%$ interval of $\left(-0.21^{\circ} \mathrm{C},-0.10^{\circ} \mathrm{C}\right)$ per decade. This is a surprising result because one would expect an increase in temperature due to climate change. Figure 6 shows a plot of the temporal-trend regression coefficient $(\% /$ year $)$ obtained by the Sen method $[45,46]$ for the summer extreme 2 meters-height temperature in the ERA Interim reanalysis used to externally forcing the WRF model in the study period 1981-2015. ERA Interim reanalysis shows a predominant positive trend for the study period (1981-2015), mainly in the central area of the Iberian Peninsula. However, this positive trend tends to decrease towards the west of the IP, with some regions showing, although small, a negative trend. Also, from the figure we can see that the WRF model shows a negative trend throughout the west, including the study region, and a positive trend in the center of the IP. It is possible, however, that internal and model variability in the WRF model which persist after the constraint of the boundary conditions given by the external reanalysis $[47,48]$ lead to the enhanced, although small, 
Trend Tmax summer (ERAIN)

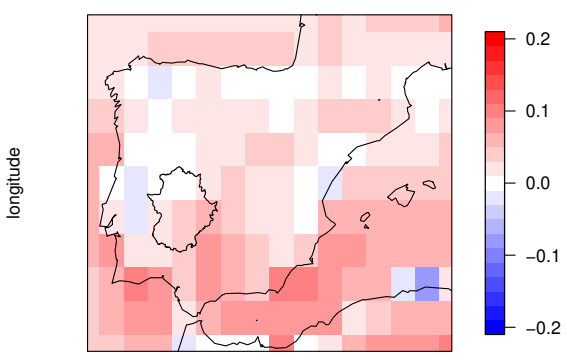

latitude
Trend Tmax summer (WRF)

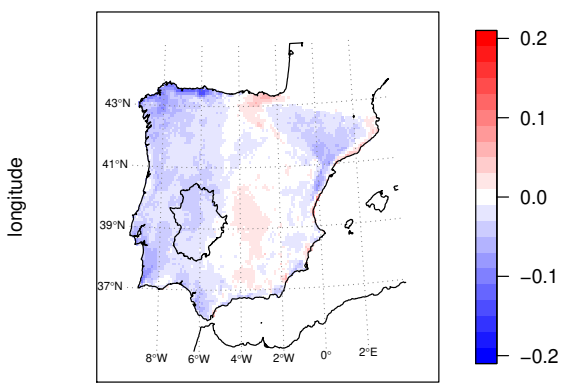

latitude

Fig. 6 Temporal-trend regression coefficient $\left({ }^{\circ} \mathrm{C} /\right.$ year $)$ obtained by the Sen method for summer extreme 2 meters-height temperature in ERA Interim reanalysis (left) and in WRF model (right).

negative trends in extreme temperatures appearing in a simulation as short as that of the present work (35 years). See, for example, [49], [50], [51] for studies of temporal trends in RCM simulations.

An interesting parameter to study is the shape parameter, which provides information of the shape of the extreme temperature probability distribution function (PDF). A positive value indicates that there is no upper bound on the extreme temperatures, and a negative value that there is an upper bound. Figure 7 shows the posterior distribution of this parameter for the chosen model. The mean $(5 \%, 95 \%)$ is $-0.994(-0.092,-0.106)$, thus supporting a bound

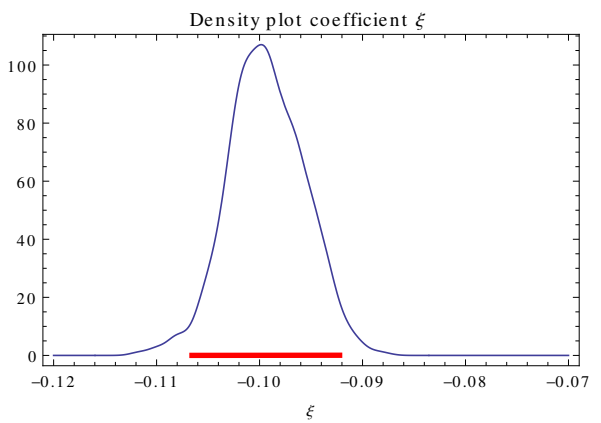

Fig. 7 Shape parameter $\xi$. The red line shows the interval $(5 \%, 95 \%)$.

on the extreme temperatures for the region under study. The negative value of the shape parameter in the GEV and Generalized Pareto Distribution used to fit the extreme temperature distribution seems to be a universal feature because several other workers have found similar negative values for different parts of the world (see, for example, [52] in Malaysia, [53] in Mexico, [54] in 


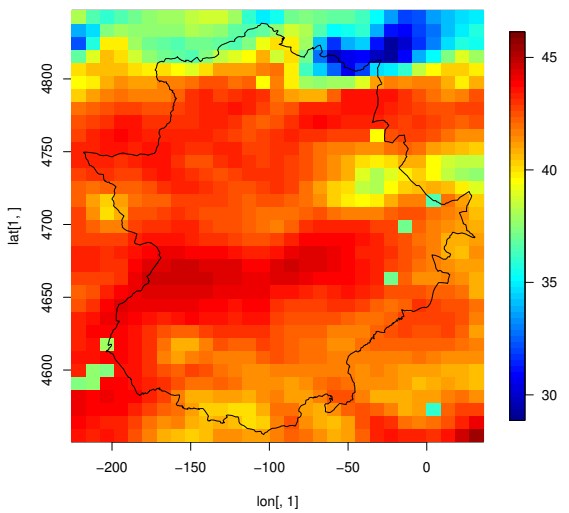
in France). to those just shown for the $3 \times 3$ subsampling. level is beginning of the period,

considering the normalization used in $f(t)$.

USA, [55] for different parts of the world, [56] for the North Atlantic, and [57]

It is important to note that the calculations with the model have also been performed with other subsampling $(2 \times 2,4 \times 4)$, obtaining results similar

Once the parameters of the GEV distribution are known, one could display maps of them. From the perspective of field practitioners, however, it would be more interesting to combine them and display return level maps. The Tyear return level is the high quantile for which the probability that the annual maximum exceeds this quantile is $1 / \mathrm{T}$ (see [58] and [16] for a definition of the T-year return level and T-year return period in a non-stationary framework). From the GEV distribution, one gets the expression

$$
y_{p}(s)=\mu_{s}-\frac{\sigma_{s}}{\xi_{s}}\left(1-(-\log (1-p))^{-\xi_{s}}\right),
$$

with $p=1 / T$. For the non-stationary model (Equation (7)), the T-year return

$$
y_{p}(s, t)=\mu_{0 s}+\mu_{1 s} \cdot f(t)-\frac{\sigma_{s}}{\xi_{s}}\left(1-(-\log (1-p))^{-\xi_{s}}\right) .
$$

From this equation, one can evaluate the difference between the end and the

$$
\Delta y_{p}(s)=2 \mu_{1 s},
$$

Fig. 8 Mean of the 40-year return level for $\mathrm{t}=0$ (left), and $\Delta y_{0.05}$ (right).

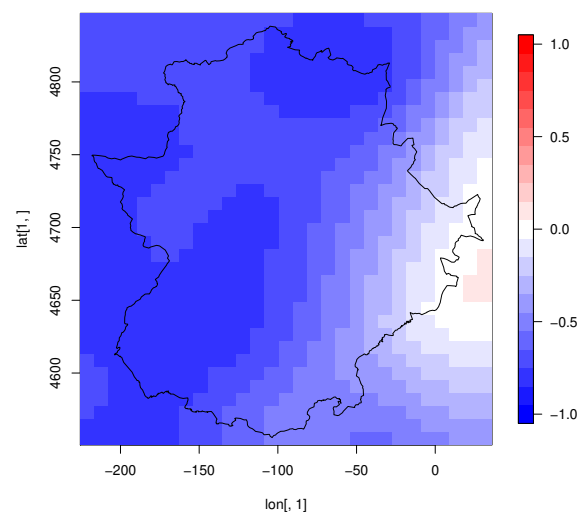

Figure 8 (left) shows a map of the 40-year (97.5 percentile) return level. As may be appreciated by comparing this figure with that of the topography (2), the spatial distribution of the 40-year return level is completely determined 
by the topography as should be expected for a variable such as temperature. We also calculated the difference in the 40-year return level between the end and the beginning of the study period. The resulting map is shown in Figure 8 (right). One sees that, for most of the region, there is a decrease in the return level by about $0.8^{\circ} \mathrm{C}$ to $1.0^{\circ} \mathrm{C}$, except at the eastern edge of the region where the decrease approaches $0^{\circ} \mathrm{C}$

One of the advantages of having a spatial model such as that used in this work is the possibility of predicting the GEV distribution parameters at a non-observed site. In this sense, we evaluated the T-year return period for a set of 28 weather stations in the region under study. To obtain values of the location and scale parameters, we drew from a normal distribution with mean and variance given by Equations (22) and (23), and with Equation (34) we got the T-year return period. Figure 9 shows a scatter plot of the predicted versus observed 40-year return levels, together with the regression line through the origin $y \sim x$ (blue) and the 1:1 line (black). The observed 40-year return level was obtained by fitting a GEV model to each observed data set using the $\mathrm{R}$ package ismev ([59]). The regression coefficient is $0.96( \pm 0.014)$, the bias (observed - predicted) is $1.66^{\circ} \mathrm{C}$, and the RMS is $2.30^{\circ} \mathrm{C}$. The results seem quite promising, although the predicted values are underestimates of those observed.

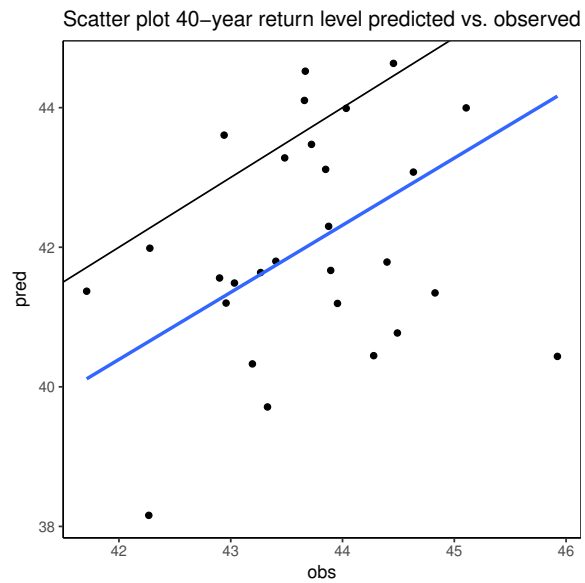

Fig. 9 Scatter plot of the predicted and 'observed' 40-year return levels for 28 observatories in the region. The black line is the 1:1 line, and the blue line the $y \sim x$ linear regression.

\section{Summary and Conclusions}

In the present work, we have described a two-step study of extreme temperatures in the region of Extremadura (Spain). In the first step, we used the WRF model to obtain a set of extreme temperatures for the period 1981-2015 in our region. The boundary conditions needed by the model were obtained 
from ECMWF's ERA Interim data. In the second step, a statistical study was made of the extreme temperature data obtained in the region. To this end, a Bayesian hierarchical spatio-temporal model with a GEV parametrization of the extreme event data was used. Two advantages of this kind of model are that it reduces the uncertainty in the parameters of the statistical distribution by pooling data among 'observatories' in a consistent way, and that it can project important features of the statistical distribution, such as the T-year return level, to non-observing sites.

The spatial-trend parameter given by the model chosen was quite consistent with that corresponding to the dry adiabatic gradient, i.e., the gradient one would expect in the summer season when adiabatic conditions are prevalent in the atmospheric boundary layer. The negative trend in the location parameter is consistent with the negative trend that the WRF model shows for the extreme temperature in the western part of the Iberian Peninsula which has enhanced the trend given by ERA Interim for the same region. A possible reason for this result may lie in the internal and model uncertainties in using the WRF model. The shape parameter of the GEV model was negative, showing that there is an upper bound to the extreme temperatures over the region.

An important product we have obtained from the statistical model is that of obtaining return levels at non-observing sites. A comparison of the 40-year return levels of observed temperatures with those predicted by the statistical model showed a bias (observed minus predicted) of $1.66^{\circ} \mathrm{C}$.

Acknowledgments. Thanks are due to the Spanish State Meteorological Agency for providing the daily temperature time series used in this study.

Funding. This project has been funded by Junta de ExtremaduraConsejería de Economía e Infraestructuras (FEDER, Proyecto IB16063) and by Junta de Extremadura-Research Group Grants (GR18097).

Author contributions. All the authors contributed equally to this work. All authors have read and agreed to the published version of the manuscript.

\section{References}

[1] Cubasch, U., Wuebbles, D., Chen, D., Facchini, M.C., Frame, D., Mahowald, N., Winther, J.-G.: Introduction. In: Stocker, T.F., Qin, D., Plattner, G.-K., Tignor, M., Allen, S.K., Boschung, J., Nauels, A., Xia, Y., Bex, v., Midgley, P.M. (eds.) Climate Change 2013: The Physical Science Basis. Contribution of Working Group I to the Fifth Assessment Report of the Intergovernmental Panel on Climate Change. Cambridge University Press, Cambridge, UK and NY USA (2013)

[2] Field, C.B., Barros, V., Stocker, T.F., Qin, D., Dokken, D.J., Ebi, K.L., Mastrandrea, M.D., Mach, K.J., Plattner, G.-K., Allen, S.K., Tignor, M., Midgley, P.M.e..: Managing the Risks of Extreme Events and Disasters 
to Advance Climate Change Adaptation. Cambridge University Press, Cambridge, UK and NY USA (2012)

[3] García-Herrera, R., Díaz, J., Trigo, R.M., Luterbacher, J., Fischer, E.M.: A Review of the European Summer Heat Wave of 2003. Critical Reviews in Environmental Science and Technology 40(4), 267-306 (2010). https: //doi.org/10.1080/10643380802238137

[4] Barriopedro, D., Fischer, E.M., Luterbacher, J., Trigo, R.M., GarcíaHerrera, R.: The hot summer of 2010: redrawing the temperature record map of Europe. Science 332, 220-224 (2011). https://doi.org/10.1126/ science. 1201224

[5] Zhang, X., Alexander, L., Hegerl, G.C., Jones, P., Tank, A.K., Peterson, T.C., Trewin, B., Zwiers, F.W.: Indices for monitoring changes in extremes based on daily temperature and precipitation data. WIREs Climate Change 2(6), 851-870 (2010). https://doi.org/10.1002/wcc.147

[6] Peterson, T.C., Folland, C.K., Gruza, G., Hogg, W.D., Mokssit, A., Plummer, N.: Report on the Activities of theWorking Group on Climate Change Detection and Related Rapporteurs. WCDMP-47, WMO-TD 1071, WMO (2001)

[7] Peterson, T.C.: Climate change indices. Bulletin of the World Meteorological Organization 54, 83-86 (2005)

[8] Davison, A.C., Padoan, S.A., Ribatet, M.: Statistical modeling of spatial extremes. Statistical Science 27(2), 161-186 (2012). https://doi.org/10. 1214/11-STS376

[9] Casson, E., Coles, S.: Spatial Regression Models for Extremes. Extremes $\mathbf{1}(4), 449-468$ (1999)

[10] Cooley, D., Nychka, D., Naveau, P.: Bayesian spatial modeling of extreme precipitation return levels. Journal of the American Statistical Association 102(479), 824-840 (2007)

[11] Schliep, E.M., Cooley, D., Sain, S.R., Hoeting, J.: A comparison study of extreme precipitation from six different regional climate models via spatial hierarchical modeling. Extremes 13, 219-239 (2010). https://doi. org/10.1007/s10687-009-0098-2

[12] Renard, B.: A Bayesian hierarchical approach to regional frequency analysis. Water Resources Research 47, 11513 (2011). https://doi.org/10.1029/ 2010WR010089

[13] Gelfand, A.E., Zhu, L., Bradley, P.C.: On the Change of Support Problem 
for Spatio-Temporal Data. Biostatistics 2(1), 31-45 (2001)

[14] Fuentes, M., Guttorp, P., Challenor, P.: Statistical Assessment of Numerical Models. International Statistical Review 71(2), 201-222 (2003)

[15] Epstein, E.S.: Statistical Inference and Prediction in Climatology: A Bayesian Approach. Meteorological Monographs, vol. 20. American Meteorological Society, Boston (1985)

[16] Kharin, V.V., Zwiers, F.W.: Estimating extremes in transient climate change simulations. Journal of Climate 18, 1156-1173 (2005)

[17] Tomassini, L., Jacob, D.: Spatial analysis of trends in extreme precipitation events in high-resolution climate model results and observations for germany. J. Geophysical Reseach 114, 12113 (2009). https://doi.org/10. 1029/2008JD010652

[18] Craigmile, P.F., Guttorp, P.: Can regional climate model reproduce observed extreme temperatures ? Statistica 73, 103-122 (2013)

[19] Skamarock, W.C., Klemp, J.B., Dudhia, J., Gill, D.O., Barker, D., Duda, M.G., Huang, X.-Y., Wang, W., Powers, J.G.: A Description of the Advanced Research WRF Version 3. Technical Note NCAR/TN475+STR, University Corporation for Atmospheric Research. (2008). https://doi.org/10.5065/D68S4MVH

[20] Dee, D.P., Uppala, S.M., Simmons, A.J., Berrisford, P., Poli, P., Kobayashi, S., Andrae, U., Balmaseda, M.A., Balsamo, G., Bauer, P., Bechtold, P., Beljaars, A.C.M., van de Berg, L., Bidlot, J., Bormann, N., Delsol, C., Dragani, R., Fuentes, M., Geer, A.J., Haimberger, L., Healy, S.B., Hersbach, H., Holm, E.V., Isaksen, L., Kallberg, P., Kohler, M., Matricardi, M., McNally, A.P., Monge-Sanz, B.M., Morcrette, J.-J., Park, B.-K., Peubey, C., de Rosnay, P., Tavolato, C., Thépaut, J.-N., Vitart, F.: The ERA-Interim reanalysis: configuration and performance of the data assimilation system. Quarterly Journal of the Royal Meteorological Society 137(656), 553-597 (2011). https://doi.org/10.1002/qj.828

[21] Hong, S.-Y., Lim, J.O.J.: The WRF Single moment 6-Class Microphysics Scheme (WSM6). Journal of the Korean Meteorological Society 42(2), 129-151 (2006). https://doi.org/10.1175/MWR3199.1

[22] Iacono, M.J., Delamere, J.S., Mlawer, E.J., Shephard, M.W., Clough, S.A., Collins, W.D.: Radiative forcing by long-lived greenhouse gases: Calculations with the AER radiative transfer models. J. Geophys. Res. 113, 13103 (2008). https://doi.org/10.1029/2008JD009944.

[23] Jiménez, P.A., Dudhia, J., González-Rouco, J.F., Navarro, J., Montávez, 
J.P., García-Bustamente, E.: A Revised Scheme for the WRF Surface Layer Formulation. Monthly Weather Review 140(3), 898-918 (2012). https://doi.org/0.1175/MWR-D-11-00056.1

[24] Hong, S.-Y., Noh, Y., Dudhia, J.: A new vertical diffusion package with an explicit treatment of entrainment processes. Monthly Weather Review 139(9), 2318-2341 (2006). https://doi.org/10.1175/MWR3199.1

[25] Hong, S.-Y.: A new stable boundary-layer mixing scheme and its impact on the simulated East Asian summer monsoon. Q.J.R. Meteorol. Soc 136(1481-1496) (2010). https://doi.org/10.1002/qj.665

[26] Chen, F., Dudhia, J.: Coupling an advanced land surface-hydrology model with the Penn State-NCAR MM5 modeling system. Part I: Model implementation and sensitivity. Monthly Weather Review 129, 569-585 (2001). https://doi.org/10.1175/1520-0493(2001)129〈0569:CAALSH $\rangle 2.0 . C O ; 2$

[27] Grell, G.A., Freitas, S.R.: A scale and aerosol aware stochastic convective parameterization for weather and air quality modeling. Atmos. Chem. Phys 14, 5233-5250 (2014). https://doi.org/10.5194/acp-14-5233-2014

[28] Fita, L., Polcher, J., Giannaros, T.M., Lorenz, T., Milovac, J., Sofiadis, G., Katragkou, E., Bastin, S.: CORDEX-WRF v1.3: development of a module for the Weather Research and Forecasting (WRF) model to support the CORDEX community. Geoscientific Model Development 12(3), 1029-1066 (2019). https://doi.org/10.5194/gmd-12-1029-2019

[29] Berliner, M.L.: Hierarchical Bayesian time series models. In: Hanson, K.M., Silver, R.N. (eds.) Maximum Entropy and Bayesian Methods. Fundamental Theories of Physics, vol. 79. Springer, ??? (1996)

[30] Cressie, N., Wikle, C.K.: Statistics for Spatio-Temporal Data. Wiley, ??? (2011)

[31] Gilks, W.R., Richardson, S., Spiegelhalter, D.J.: Introducing Markov Chain Monte Carlo. In: Gilks, W.R., Richardson, S., Spiegelhalter, D.J. (eds.) Markov Chain Monte Carlo in Practice. Chapman \& Hall, ??? (1996)

[32] Berliner, L.M.: Physical-statistical modeling in geophysics. Journal of Geophysical Research: Atmospheres 108(D24) (2003). https://doi.org/ 10.1029/2002JD002865

[33] Banerjee, S., Gelfand, A.E., Finley, A.O., Sang, H.: Gaussian predictive process models for large spatial data sets. J. Royal Statistical Society, B $\mathbf{7 0}(4), 825-848(2008)$ 
[34] Finley, A.O., Sang, H., Banerjee, S., Gelfand, A.E.: Improving the performance of predictive process modeling for large datasets. Computationl Statistics and Data Analysis 53, 2873-2884 (2009)

[35] Eidsvik, J., Finley, A.O., Banerjee, S., Rue, H.: Approximate bayesian inference for large spatial datasets using predictive process models. Computationl Statistics and Data Analysis 56, 1362-1380 (2012)

[36] Cowles, M.K., Carlin, B.P.: Markov Chain Monte Carlo Convergence Diagnostics: A Comparative Review. Journal of the American Statistical Association 91(434), 883-904 (1996)

[37] Finley, A.O., Banerjee, S., Gelfand, A.E.: spBayes for Large Univariate and Multivariate Point-Referenced Spatio-Temporal Data Models. Journal of Statistical Software 63(13), 1-28 (2015)

[38] Gelman, A., Hwang, J., Vehtari, A.: Understanding predcitive information criteria for bayesian models. Statistics and Computing 24, 997-1016 (2014). https://doi.org/10.1007/s11222-013-9416-2

[39] Lynch, S.M., Bruce, W.: Bayesian posterior predictive checks for complex models. Sociological Methods \& Research 32(3), 301-335 (2004). https: //doi.org/10.1177/0049124103257303

[40] Gelman, A., Meng, X.-L., Stern, H.: Posterior predictive assessment of model fitness via realized discrepancies (with discussion). Statistica Sinica 6, 733-807 (1996)

[41] Serrano-Notivoli, R., Beguería, S., de Luis, M.: Stead: a high-resolution daily gridded temperature dataset for spain. Earth System Science Data 11(3), 1171-1188 (2019). https://doi.org/10.5194/essd-11-1171-2019

[42] Sang, H., Gelfand, A.E.: Hierarchical modeling for extreme values observed over space and time. Environ. Ecol. Stat. 16, 407-426 (2009). https://doi.org/10.1007/s10651-007-0078-0

[43] Cooley, D., Sain, S.R.: Spatial Hierarchical Modeling of Precipitation Extremes From a Regional Climate Model. Journal of Agricultural, Biological, and Environmental Statistics 15(3), 381-402 (2010)

[44] Gallego, M.C., Trigo, R.M., Vaquero, J.M., Brunet, M., García, J.A., Sigró, J., Valente, M.A.: Trends in frequency indices of daily precipitation over the Iberian Peninsula during the last century. J. Geophysical Reseach 116, 02109 (2011). https://doi.org/10.1029/2010JD014255

[45] Sen, P.K.: Estimates of the regression coefficient based on kendall's tau. Journal of the American Statistical Association 63, 1379-1389 (1968) 
[46] Hirsch, R.M., Smith, R.A.: Techniques of trend analysis for monthly water quality data. Water Resources Research 18, 107-121 (1982)

[47] Alexandru, A., Elia, R., Laprise, R., Separovic, L., Biner, S.: Sensitivity study of regional climate model simulations to large-scale nudging parameters. Monthly Weather Review 137, 1666-1686 (2009). https: //doi.org/10.1175/2008MWR2620.1

[48] Hawkins, E., Sutton, R.: The potential to narrow uncertainty in regional climate predictions. Bulletin of the American Meteorological Society 90, 1095-1107 (2009). https://doi.org/10.1175/2009BAMS2607.1

[49] Lorenz, P., Jacob, D.: Validationof temperature trends in the ENSEMBLES regional climate model runs driven by ERA40. Climate Research 44, 167-177 (2010). https://doi.org/10.3354/cr00973

[50] Bukovsky, M.S.: Temperature trend in the NARCACAP regional climate models. Journal of Climate 25, 3985-3991 (2012). https://doi.org/10. 1175/JCLI-D-11-00588.1

[51] Huth, R., Miksovsky, J., Stepanek, P., Belda, M., Farda, A., Chladova, Z., Pisoft, P.: Comparative validation os statistical and dynamical downscaling models on a dense grid in central Europe: temperature. Theoretical and Applied Climatology 120, 533-553 (2015). https://doi.org/10.1007/ s00704-014-1190-3

[52] Salleh, N.H.M., Hasan, H.: Generalized Pareto Distribution for Extreme Temperatures in Peninsular Malaysia . Science International(Lahore) 30, 63-67 (2018)

[53] García-Cueto, O.R., Santillan-Soto, N., Quintero-Muñoz, M., OjedaBenitez, S., Velázquez-Limon, N.: Extreme temperature scenarios in mexicali, mexico under climate change conditions. Atmosfera 26, 509-520 (2013)

[54] Furrier, E.M., Katz, R.W., D., W.M., Furrer, R.: Statistical modeling of hot spells and heat waves. Climate Research 43, 191-205 (2010)

[55] Brown, S.J., Caesar, J., Ferro, C.A.T.: Global changes in extreme daily temperature since 1950. J. Geophysical Reseach 113, 05115 (2008). https: //doi.org/10.1029/2006JD008091

[56] Nogaj, M., Yiou, P., Parey, S., Malek, F., Naveau, P.: Amplitude and frequency of temperature extremes over the North Atlantic region. Geophysical Research Letters 33(L10801) (2006). https://doi.org/10.1029/ 2005GL024251 
[57] Parey, S., Malek, F., Laurent, C., Dacunha-Castelle, D.: Trends and climate evolution: Statistical approach for very high temperatures in france. Climatic Change 81, 331-352 (2007). https://doi.org/10.1007/ s10584-006-9116-4

[58] Cooley, D.: Return periods and return levels under climate change. In: AghaKouchak, A., Easterling, D., Hsu, K., Schubert, S., Sorooshian, S. (eds.) Extremes in a Changing Climate, pp. 97-114. Springer, Dordrecht (2013). https://doi.org/10.1007/978-94-007-4479-0

[59] Coles, S.: An Introduction to Statistical Modeling of Extreme Values, p. 208. Springer, London (2001) 